\title{
Desafíos para una ciudadanía inclusiva: competencia digital entre adultos mayores y jóvenes
}

\section{Challenges for inclusive citizenship: digital literacy between elderly and young}

\author{
Ana Amaro Agudol \\ Erika González García ${ }^{2}$ \\ Nazaret Martínez-Heredia ${ }^{3}$
}

Resumo: En esta investigación se estudiarán las competencias digitales tanto en mayores como en jóvenes universitarios. El objetivo se basa en comparar descriptivamente la competencia digital en conocimiento y uso de las TIC en la comunicación social y aprendizaje colaborativo, competencias de uso para la búsqueda y tratamiento de la información, así como las competencias interpersonales de su uso entre jóvenes y mayores de la Facultad de Ciencias de la Educación de la Universidad de Granada (España). Se ha utilizado una metodología descriptiva de corte cuantitativo utilizando el cuestionario. Los resultados arrojan una enorme diferencia en torno a las competencias estudiadas entre ambas generaciones.

Palavras-Chave: ciudadanía; inclusión; competencia digital; adultos mayores; jóvenes.

\footnotetext{
1 Universidad de Granada (UGR). Granada, España.

https://orcid.org/0000-0001-8033-0488 E-mail: anaamaro@ugr.es

2 Universidad de Granada (UGR). Granada, España.

https://orcid.org/0000-0003-2465-7288 E-mail: erikag@ugr.es

3 Universidad de Granada (UGR). Granada, España.

https://orcid.org/0000-0001-7029-7127 E-mail: nazareth@ugr.es
}

Abstract: In this research the digital competences will be studied so much in major and young persons in university. The aim of our research is to compare 
descriptively the digital competence in knowledge and use of the TIC in the social communication and collaborative learning, competences of use for the search and data processing, as well as the interpersonal competences of their use between young and major of the Faculty of Educational Sciences at University of Granada (Spain). There has been in use a descriptive quantitative methodology using the questionnaire as tool to obtain information. The results throw an enormous difference to the competences studied between both generations.

Keywords: citizenship; inclusion; digital literacy; seniors; young people. 


\section{Introducción}

No cabe duda del auge e importancia que están teniendo en la actualidad los estudios en torno al tratamiento y uso de las Tecnologías de la Información y la Comunicación (TIC). Éstas han tomado un papel primordial en la interactividad, virtualidad y flujo de información a todos los niveles y edades, tanto en el ámbito laboral como en el interpersonal. En este contexto de auge tecnológico, consideramos de suma importancia estudiar el uso que se hace de Internet, las competencias 2.0 de los estudiantes universitarios jóvenes y personas mayores y qué problemáticas se nos plantean.

En el informe de investigación que presentamos concurren dos vías de disertación: por un lado, el uso de Internet y las competencias 2.0 en jóvenes y mayores universitarios y, por otro, el estudio empírico realizado a través de un cuestionario sobre la temática que abordaremos a lo largo del artículo.

La sociedad actual se caracteriza, entre otras razones, porque nunca antes tanto la información como el conocimiento habían estado al alcance de la mayor parte de la población mundial, aunque somos conscientes de que aún queda mucho camino por recorrer, pues sigue existiendo una enorme brecha digital no sólo entre generaciones sino también entre diferentes países que están menos desarrollados en cuanto al acceso a las tecnologías. Por otro lado, teniendo una serie de competencias digitales y los recursos tecnológicos necesarios, cualquier ciudadano puede comunicarse (GONZÁLEZ-REYES, 2009), acceder desde cualquier lugar del mundo e incluso autoformarse. Por medio de Internet podemos realizar gran parte de las actividades diarias que nos facilitaban otras tecnologías (HEREDIA y GARCÍA, 2017; DOMÍNGUEZ, 2009; ÁLVAREZ, 2011; MARTÍNEZ, CABECINHAS y LOSCERTALES, 2011; DAGHAN, 2017). En este aspecto y de acuerdo con Castells (2000), la Sociedad de la Información supone una nueva revolución industrial. En esta panorámica de conexiones/desconexiones virtuales cohabitan dos generaciones diferentes; la llamada generación "before computer" (FREIXA, 2006) y aquella que ha nacido y crecido 
interactuando con múltiples dispositivos tecnológicos, los denominados "nativos/as digitales".

\section{Marco Conceptual}

\section{Uso de internet e inclusión social}

El uso de los equipos tecnológicos por parte de la población es cada vez más alto, para formarse, comunicarse, trabajar o distraerse. Internet no se limita a la mera transmisión de información, sino que se convierte en un poderoso mecanismo de socialización, de transmisor de ideas y de valores (FAINHOLC, 2006; XAVIER y CABECINHAS, 2000). La sociedad de la información y el conocimiento exige una alfabetización digital de sus ciudadanos cada vez mayor. La educación, en ámbitos formales e informales, tiene un rol destacable al intentar favorecer la inclusión y la inserción social, puesto que ayuda a desarrollar competencias que permiten acceder, a los contenidos en la red, de manera autónoma, crítica y responsable y para ello los gobiernos deben ser facilitadores de los recursos necesarios ( $\square$ AHIN, 2018; CANTABRANA, ESTEBANELL y TEDESCO, 2015). La finalidad de su aparición fue alcanzar información segura, rápida y económica para facilitar la comunicación, hoy se ha convertido en un medio que puede ser causa de cambios significativos en las personas y en la sociedad.

Estudios recientes lanzan datos evidentes en torno al papel crucial que tienen las tecnologías como mediadoras emocionales que sientan bases estratégicas y estructurales de relaciones significativas, enriqueciendo las líneas emergentes de debate existentes en torno a la aplicación de las Tecnologías de la Información y la Comunicación en el desarrollo socioafectivo (COLÁS-BRAVO, GONZÁLEZ-RAMÍREZ y DE PABLOS-PONS, 2013; ESPINOZA, 2015; CALVO y SAN FABIÁN, 2018).

En el año 2016, a nivel mundial, 3.500 millones de personas estaban utilizando Internet, de los cuales 2,5 millones eran de países en desarrollo (ITU, 2017). En consecuencia, consideramos que toda nuestra 
actividad está ligada de un modo u otro al mundo digital, hasta tal punto que se vuelve impensable afrontar la vida sin estas nuevas herramientas. Esta oleada tecnológica no está exenta de flaquezas y riesgos, se manifiesta en un mundo donde existe una gran brecha entre las diferentes partes del planeta, desigualdad en cuanto a sexo, edad, cultura, etc. De acuerdo con esta premisa, se han llevado a cabo numerosos estudios con el objetivo de analizar la influencia de Internet en los más jóvenes, por considerarlos un sector prioritario y más vulnerable. De este modo, se ha intentado conocer tanto los efectos positivos o negativos que Internet pueda tener, como los usos que se realizan (LIVINGSTONE y HELSPER, 2010; YANG y TUNG, 2007; RUÍZ-CORBELLA y DE JUANAS, 2013; BALLESTEROS y MEGÍAS, 2015).

En el panorama español y según fuente del Instituto Nacional de Estadística (2017), el 84,6\% de la población de 16 a 74 años ha usado Internet en los tres últimos meses. El 69,0\% lo hace a diario, este porcentaje es ligeramente superior ( 1,3 puntos) al del año pasado. Las persona de entre 65 y 74 años que usan Internet son un 46.5\%, frente a los jóvenes de entre 16 a 24 años que usa Internet un $98.0 \%$, es decir más del doble que las personas más mayores. Pese a estos resultados, tal y como muestra el estudio realizado por Linne (2015), se pone de manifiesto que los estudiantes universitarios españoles no son adictos, a su juicio, de Internet y más concretamente de las redes sociales. Investigaciones recientes (LLORENTE, VIÑARÁS y SÁNCHEZ, 2015; MARTÍNEZ, CABECINHAS y LOSCERTALES, 2011), muestran que las personas mayores universitarias se conectan a Internet frecuentemente, a diario o entre dos o tres veces por semana. Destacan la importancia que tiene Internet para estar actualizados, para contactar con la familia y los amigos, para el uso académico y para consultar la prensa. Consideran que la red es de fácil uso pero podrían vivir sin ella. Es decir, no es algo imprescindible en su vida como pueda serlo para los jóvenes universitarios.

El progresivo envejecimiento de las sociedades ha llevado a los organismos internacionales y europeos a desarrollar programas de envejecimiento activo, capaces de construir una nueva cultura sobre el 
papel de las personas mayores en la sociedad (LLORENTE, VIÑARÁS y SÁNCHEZ, 2015).

Los jóvenes son los principales motores de la sociedad del futuro, pero no por ello debemos dejar de prestar atención al colectivo de personas mayores, la tasa de personas mayores se incrementa en todo el mundo, y en el caso particular de España su evolución representa un importante porcentaje no solo de la población actual sino también de la futura. "La proporción de población de 65 años y más ha pasado de representar un $11,2 \%$ en 1981 a hacerlo con un 17,3\% veinte años después y con un $18,7 \%$ en 2015. Pero, si se traduce a efectivos, supone que entre 1981 y 2015 la población anciana se ha duplicado en algo más de 200.000 individuos. Entre el 2050 y el 2060, la población total bajará en algo más de dos millones de habitantes y, de ellos, el grupo de los mayores de 65 años solo perderá el 0,1\%. En el año 2060 habrá algo menos de 15 millones de mayores, menos del doble que en la actualidad, y representarán más de un tercio del total de la población española $(35,6 \%)$ " (Instituto de Mayores y Servicios Sociales, IMSERSO, 2017, p. 36).

Las TIC tienen una vertiente muy positiva, ya que, las competencias mediáticas pueden convertirse en herramientas para mejorar la vida social. Para las personas mayores las actividades que se pueden llevar a cabo son múltiples y útiles para fomentar la creatividad, practicar la escritura, mejorar la sociabilidad, ejercitar la memoria y la mente, aprender cosas que no han podido antes debido a la falta de tiempo, su profesión, etc. La Unión Europea declaró el año 2012 como el “Año Europeo del Envejecimiento Activo y de la Solidaridad Intergeneracional” para combatir el efecto del envejecimiento demográfico sobre los modelos sociales de los Estados miembros y promover la creación de una cultura del envejecimiento activo como un proceso permanente en una sociedad multiedad (LLORENTE, VIÑARÁS y SÁNCHEZ, 2015). Hay una mejora evidente de la vida social ya que el hecho de que una persona mayor goce de un buen estado de bienestar supondrá estar sano física, emocional y psicosocialmente. Todo esto tiene una finalidad, la de inclusión social ya que la falta de competencias digitales es causa de 
exclusión social entendiéndose ésta como aquello que impide el pleno desarrollo de las personas según sus verdaderos deseos y capacidades (DUQUE, 2016; PAVON, 2000; ROMÁN, ALMANSA y CRUZ, 2016).

La sociedad de la información y el conocimiento exige una alfabetización digital de sus ciudadanos cada vez mayor. La enseñanza de la ciudadanía debería ir más allá de los comportamientos éticos y llegar a la raíz de la conducta humana, cultivando hábitos relacionados con la inteligencia y la voluntad para que la toma de decisiones realmente esté orientada hacia el bien individual y colectivo.

En el ámbito formal e informal, la educación tiene un rol destacable al intentar favorecer la inclusión y la inserción social. Ayuda a desarrollar las competencias que permiten acceder, registrar, editar, publicar y compartir contenidos en la red, de manera autónoma, crítica y responsable. En este sentido, cabe señalar que los gobiernos deben facilitar los recursos necesarios para que esto pueda ser posible (CANTABRANA, ESTEBANELL y TEDESCO; 2015; DUQUE 2016).

En un estudio reciente (MARTÍNEZ, CABECINHAS y LOSCERTALES, 2011), se ha puesto de manifiesto los principales usos y motivaciones de los mayores activos para utilizar Internet, así como las principales barreras para aquéllos que no la utilizan. Destacan entre los usos que hacen de la misma la búsqueda de información, la actividad académica, la lectura de la prensa y también la navegación sin ningún propósito específico. Con respecto a los que no la utilizan, se debe principalmente a que les falta un empuje para hacerlo, no ven una limitación en la edad ni por la pérdida de tiempo entre otros factores.

\section{Acerca de la competencia digital}

El Parlamento europeo y el Consejo de 18 de diciembre de 2006 recogieron un conjunto de recomendaciones sobre competencias clave para el aprendizaje permanente a nivel europeo. En el mismo se debía definir las nuevas cualificaciones básicas que debe proporcionar el aprendizaje permanente como medida esencial de la respuesta de Europa ante la 
globalización y el desplazamiento hacia las economías basadas en el conocimiento, teniendo como principal baza en Europa las personas.

El concepto de Competencias nace vinculado al ámbito laboral, y hace referencia a todos aquellos aspectos (habilidades, destrezas, actitudes...) que debe poseer un profesional para desarrollar su labor de una forma eficaz.

Como consecuencia de ello, en 2006 el Parlamento Europeo y el Consejo publicaron una recomendación identificando ocho Competencias Claves para el Aprendizaje Permanente. El desarrollo de las competencias clave capacita a las personas a desarrollar al máximo sus potencialidades y habilidades innatas para poder desenvolverse en diferentes contextos a lo largo de su vida. Entre las mismas resaltar la competencia digital como base del informe de investigación que presentamos la cual implica el uso seguro y crítico de la sociedad de la información Tecnología (IST) para el trabajo, el ocio y la comunicación. Se basa en los principios básicos y habilidades en TIC: el uso de computadoras para recuperar, evaluar, almacenar, producir, presentar e intercambiar información, y comunicarse y participar en colaboración redes a través de Internet (FERRARI, 2012; BENNETT y MATON, 2010).

Según Lankshear y Knobel (2008) lo que ahora entendemos por alfabetización o competencia digital, ha evolucionado a lo largo de estas últimas décadas, desde aspectos más centrados con el acceso a la tecnología, a informaciones visuales o multimedia. La trasformación que se ha producido en los últimos años con respecto a la alfabetización o competencia digital y sobre el acceso a la tecnología es importantísima. La alfabetización digital es la conciencia, la actitud y la capacidad de las personas para utilizar adecuadamente las herramientas digitales para identificar, acceder, administrar, integrar, evaluar, analizar y sintetizar los recursos digitales, construir nuevos conocimientos, expresarse a través de los recursos multimedia y comunicarse con los demás en cualquier contexto específico de la vida (ESTEVE, 2013; GISBERT y GONZÁLEZ, 2011). 
En la actualidad los cambios vertiginosos que se están sucediendo hacen necesaria una mejora en la formación y un acceso a la misma a lo largo de la vida que, son necesarias una serie de habilidades y competencias que permitan adaptarse a una sociedad de cambios; la relación del individuo con la información ha cambiado, por ello el sector educativo plantea nuevas formas de llevar a los estudiantes a un buen desarrollo dentro de la Sociedad del Conocimiento (CHÁVEZ, CANTÚ y RODRÍGUEZ, 2016; GONZÁLEZ, ESPUNY y GISBERT, 2012).

Entendemos por tanto por competencia digital la conjunción de lo que muchos autores entienden por competencia TIC y competencia informacional. En la sociedad del conocimiento no tiene sentido hablar solo de herramientas para el almacenaje, acceso y recuperación de la información, sino que debemos trabajar, también, las habilidades y las destrezas necesarias para usar adecuadamente esta información y transformarla después en conocimiento, con el objetivo final de compartirlo. Que en definitiva es lo que va a ayudar a jóvenes y mayores a ser hábiles y competentes socialmente hablando en cualquier momento y entorno.

\section{Jóvenes y adultos mayores: competencia digital y brecha digital}

Los cambios en la sociedad han obligado a las personas a llevar a cabo un proceso obligado y necesario de formación a lo largo de la vida, como asî se recoge en el documento sobre Recomendaciones del Parlamento Europeo. Existe por tanto una necesidad real de formarse a lo largo de toda la vida y de trabajar la competencia digital, tanto en jóvenes como mayores. Según Gisbert, Espuny y González (2011, p. 76) "la competencia digital, decimos que supone la adquisición de conocimientos, destrezas y actitudes que tienen que ver con el uso elemental del hardware de los ordenadores, sus sistemas operativos como gestores del hadware, el software como herramienta de trabajo, de comunicación off-line y de comunicación on-line". 
En el momento en el que nos encontramos donde todo cambia, la competencia digital se ha convertido en algo imprescindible para afrontar los retos de la vida cotidiana de la ciudadanía, la competencia manejo o tratamiento de la información (JARAMILLO, HENNIG y RINCÓN, 2011) es fundamental para desenvolverse en la sociedad actual a la vez que resulta fundamental para el desarrollo académico y profesional de cualquier estudiante (GISBERT, ESPUNY y GONZÁLEZ, 2011).

La inclusión social juega un papel preponderante como oportunidad para humanizar la concepción de ciudadano digital, aprovechando los recursos tecnológicos para entender realidades, motivar para acercarse a los que necesitan ayuda y que son incomprendidos con frecuencia, denunciar injusticias y proponer soluciones desde los recursos multimediales que pueden ser comprendidos por una gran mayoría de personas (DUQUE, 2016).

La idea de "los nativos digitales", una generación de jóvenes conocedores de la tecnología inmersos en las tecnologías digitales que han pasado toda su vida rodeados y usando, videojuegos, reproductores de música digital, cámaras de video, teléfonos, i-pods, Internet, mensajería instantánea, mensajes de texto, multimedia y otras herramientas de la era digital que son parte integral de sus vidas ha ganado popularidad generalizada (BENNETT y MATON, 2010; GALLARDO, 2012). Investigaciones recientes han mostrado fallas en el argumento de que hay una generación identificable, o incluso un único tipo de usuario de tecnología altamente experto. Para Bullen, Morgan y Qayyum (2011), sugieren el término estudiantes digitales (digital learners) porque los estudiantes de hoy no se ajustan al estereotipo representado en el discurso de los nativos digitales. Para los autores es una cuestión social y no generacional, y sus implicaciones para la educación aún necesitan mayor estudio a profundidad.

Es necesaria una formación de los estudiantes universitarios que los prepare para un mundo cada vez más complejo y globalizado, donde la cantidad de información que tendrán que gestionar, cada día es mayor, y donde deberán utilizar herramientas tecnológicas que avanzan y 
cambian a un ritmo vertiginoso (GISBERT, ESPUNY y GONZÁLEZ, 2012).

Como recoge Abad (2014, p. 174) los datos contemplados en los Indicadores de la Agenda Digital 2011 dedicado a competencia digital muestran "que mientras el $90 \%$ de las personas entre 16 y 24 años son usuarios habituales de Internet, sólo lo son el $46 \%$ de las personas comprendidas entre los 55 y 64 años disminuyendo esta proporción al 25\% entre las personas entre 65 y 74 años. Este segmento baja especialmente al 20\% cuando se trata de personas de entre 55 y 74 años con niveles bajos de educación”.

Comprobamos de esta forma la existencia de una brecha digital de carácter generacional, entendida como las diferencias en cuanto al acceso y uso de las TIC en diferentes entornos sociales. Esta fractura entre jóvenes y mayores, generada por la discriminación en el acceso a las TIC, se ha convertido en uno de los grandes retos para la ONU y la Comisión Europea (LLORENTE, VIÑARÁS y SÁNCHEZ, 2015). Actualmente, no se ha analizado en profundidad cómo puede afectar a nuestros mayores, en su día a día, la "brecha digital". No es la tecnología la que construye la sociedad, sino que es un elemento que la caracteriza y le ayuda en sus propósitos de cambio sociocultural (ARRIDO-LORA, BUSQUEt y MUNTÉ, 2016).

La competencia digital según Hernando (2013, p. 14) “no se adquiere por "inmersión tecnológica” y es necesario aprender a aprender, pero esta capacidad es socialmente desigual y, entre otros aspectos, está ligada a la edad. La "brecha digital" además de estar relacionada con diferencias socioeconómicas también lo está, y mucho, con la edad, especialmente en grupos de edad más alejadas de las generaciones digitales como son las personas mayores de 60 años".

Una de las consecuencias más importantes del fenómeno es la modificación o inversión del proceso educativo. Si históricamente eran los adultos quienes transmitían los conocimientos, valores y costumbres a los menores, a partir de la segunda mitad del siglo XX se invierte este principio, limitándose claramente el control efectivo de los más jóvenes 
por parte de los adultos (ARRIDO-LORA, BUSQUET y MUNTÉ, 2016; HERNANDO y PHILLIPPI, 2013).

Investigaciones recientes concretan que la ciudadanía digital no viene dada exclusivamente por el conocimiento del uso de los medios, sino también por la puesta en valor de los mismos para contribuir a la mejora del entorno social (ROMÁN-GARCÍA, ALMANSA y CRUZ-DÍAZ, 2016).

El trabajo con nuestros mayores en lo que a competencia digital se refiere debe ser una prioridad y un objetivo a conseguir como eje fundamental en la educación a lo largo de la vida. La inclusión social tiene que ser entendida como una responsabilidad de los gobiernos a todas las edades y en todos los contextos.

\section{Metodología}

El objetivo principal de nuestra investigación es comparar descriptivamente la competencia digital en conocimiento y uso de las TIC en la comunicación social y aprendizaje colaborativo, competencias de uso para la búsqueda y tratamiento de la información, así como las competencias interpersonales de su uso entre jóvenes y adultos mayores de la Facultad de Ciencias de la Educación y del Aula Permanente de Formación Abierta ambas de la Universidad de Granada (España).

Para la consecución del objetivo propuesto, partiremos de una metodología cuantitativa, de corte descriptivo, la cual nos ayudará a cuantificar y analizar la información para así establecer posteriores comparaciones entre el alumnado con edades comprendidas entre 1822 años y 80-85 años.

\section{Muestra}

La investigación fue desarrollada durante el curso académico 20172018. La muestra participante de nuestro estudio ha estado formada por los alumnos del tercer curso del Grado en Educación Social de la Facultad de Ciencias de la Educación de la Universidad de Granada y 
alumnos mayores del Aula Permanente de Formación Abierta también pertenecientes a la Universidad de Granada (España). Se ha seguido un muestreo aleatorio y estratificado. El total de la población estaba compuesta por 200 estudiantes matriculados, 100 del tercer curso de Educación Social y 100 del Aula Permanente. De este modo, se obtuvo una muestra final de $n=200$ sujetos ( 119 mujeres y 81 hombres en total), los cuales 61 son mujeres y 39 hombres del tercer curso del Grado en Educación Social y, 58 mujeres y 42 hombres del Aula Permanente de Formación Abierta. Tratamos con una muestra significativa superando los sujetos necesarios de la muestra calculados a través de un intervalo de confianza del $95 \%$ y cuyos resultados precisaban la participación de, al menos, 81 estudiantes. La elección de esta población se debe, principalmente, a la experiencia de los alumnos tras haber cursado tres años en la Facultad de Ciencias de la Educación y, por tanto, poseer una madurez terminológica y una actitud crítica para ayudarnos a solventar nuestro problema de investigación, siendo éste el conocimiento de las diferencias existentes ante la competencia digital en conocimiento y uso de las TIC en la comunicación social y aprendizaje colaborativo, competencias de uso para la búsqueda y tratamiento de la información, así como las competencias interpersonales de su uso entre jóvenes y mayores en la Universidad, para poder reducir la brecha digital existente en nuestros mayores.

\section{Instrumento}

Para la recogida de información hemos utilizado un cuestionario de competencias básicas digitales adaptado del cuestionario "Competencias básicas digitales 2.0 de estudiantes universitarios” COBADI 2013 (Marca registrada: 2970648) de escala Likert (1 completamente ineficaz; 2 ineficaz; 3 eficaz; y 4 completamente eficaz) validado por medio de un juicio de expertos, donde los alumnos valorarán, por un lado, la competencia digital en conocimiento y uso de las TIC en la comunicación social y aprendizaje colaborativo, las competencias de uso para la 
búsqueda y tratamiento de la información, así como las competencias interpersonales de su uso.

La validación del cuestionario se realizó siguiendo las siguientes pautas:

1. Definición del objetivo del juicio de expertos para validar el cuestionario "Competencias Básicas Digitales".

2. Selección de cuatro expertos pertinentes teniendo en cuenta los criterios definidos anteriormente, considerando su formación académica y experiencia profesional en el área de trabajo.

3. Evaluación por medio de los expertos atendiendo a la pertinencia, cohesión, claridad y adecuación de los indicadores del cuestionario utilizando una planilla.

4. Una vez obtenidos los resultados se calculó la concordancia entre jueces y finalmente se elaboraron unas conclusiones atendiendo a la descripción psicométrica de la prueba.

Una vez realizado el juicio de expertos, el cuestionario, recoge aspectos correspondientes de las competencias básicas digitales. Posteriormente se realizó una prueba de fiabilidad mediante el alfa de Cronbach, obteniendo un índice de $\alpha=0,986$, dato que le confiere un elevado grado de coherencia $(98,6 \%)$ debido su proximidad a la unidad.

De una manera más detallada, recogemos dicha información en la siguiente tabla, donde podemos observar los ítems que corresponden a cada categoría.

Tabla 1: Cuestionario de competencias básicas digitales

\begin{tabular}{ll}
\hline COMPETENCIAS BÁSICAS DIGITALES & ÍTEMS \\
\hline Consumo de la tecnología & $1-5$ \\
\hline $\begin{array}{l}\text { Competencia digital en conocimiento y uso de las TIC } \\
\text { en la comunicación social y aprendizaje colaborativo }\end{array}$ & \\
\hline $\begin{array}{l}\text { Competencias de uso para la búsqueda y tratamiento de } \\
\text { la información }\end{array}$ & $18-29$ \\
\hline Competencias interpersonales de su uso & $30-35$ \\
\hline
\end{tabular}

Fuente: los autores 


\section{Resultados}

En este apartado exponemos los resultados obtenidos atendiendo a cada una de las dimensiones tratadas en el cuestionario, teniendo en cuenta la distinción de jóvenes y adultos mayores.

En primer lugar, respecto al consumo de la tecnología, en los jóvenes se ha obtenido un consumo total del $100 \%$, por lo que el alumnado joven posee ordenador y tablet y dispone de internet tanto en casa como en la facultad, conectándose habitualmente en casa y en la universidad. Sin embargo, con nuestros adultos mayores no ocurre lo mismo, un 30\% asume que posee medios tecnológicos e internet mayoritariamente en casa quedando un $70 \%$ restante sin conexión habitual a internet y sin poseer tablet u ordenador. En la Figura 1 podemos observar la distinción entre jóvenes y adultos mayores.

Figura 1: Consumo de la tecnología en los alumnos jóvenes vs Consumo de la tecnología en los alumnos adultos mayores

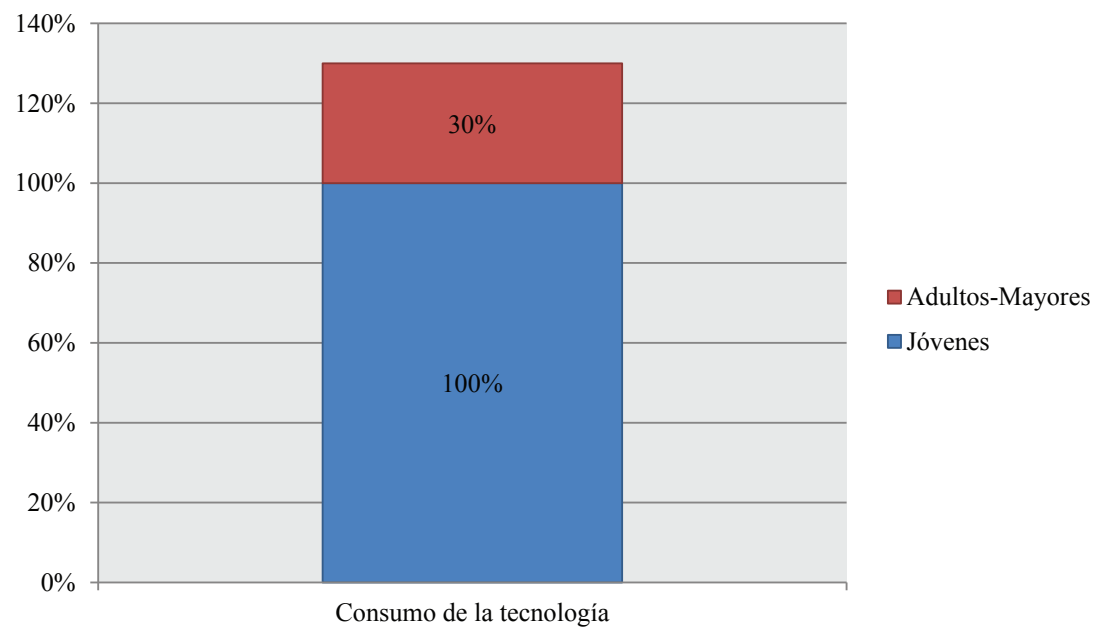

Fuente: los autores

En segundo lugar, atendiendo a la competencia digital en conocimiento y uso de las TIC en la comunicación social y aprendizaje colaborativo hemos hallado una media total de 3,66 sobre 4 en los 
alumnos de Educación Primaria y 1,50 sobre 4 en los alumnos mayores, siendo el ítem 7 (uso de la mensajería instantánea como herramienta principal de comunicación con otras personas) al que más puntuación se le otorga en ambos estudios. Sin embargo los jóvenes otorgan una menor puntuación al ítem 16 haciendo referencia al uso de marcadores sociales. Nuestros mayores otorgan una menor puntuación al ítem 8 (puedo comunicarme por redes sociales) y al 17 (capacidad para utilizar plataformas de educación). Por lo que podemos decir que nuestros jóvenes poseen una competencia digital atendiendo al uso y conocimiento de las TIC bastante elevada, en cambio, nuestros mayores la poseen en menor medida.

Figura 2: Competencia digital en conocimiento y uso de las TIC en la comunicación social y aprendizaje colaborativo. Adultos mayores VS Jóvenes.

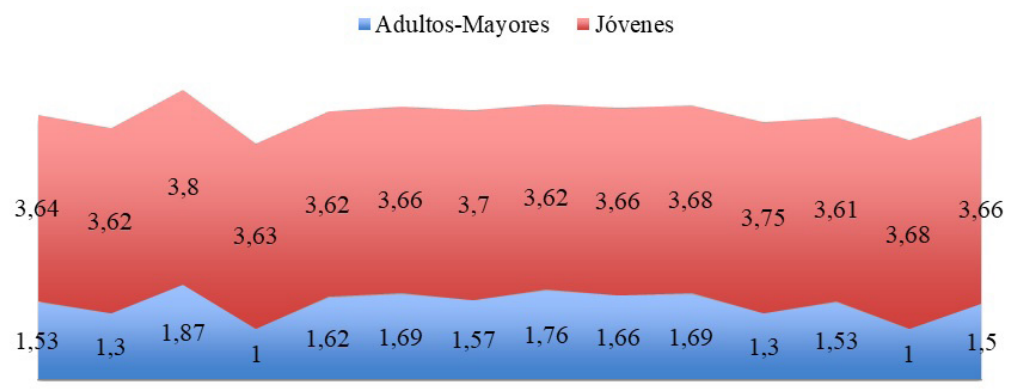

ítem5 item6 item7 item8 item9 ítem 10 ítem 11 ítem 12 ítem 13 ítem 14 ítem 15 ítem 16 ítem 17 Media

Fuente: los autores

En tercer lugar, haciendo referencia a las competencias de uso para la búsqueda y tratamiento de la información hemos hallado una media total de 3,52 sobre 4 en los alumnos de Educación Primaria y 2,66 sobre 4 en los alumnos mayores. En ambos colectivos, al ítem 18 (navegar por internet con diferentes navegadoras) es el que más puntuación se le ha otorgado. Sin embargo, otorgan una menor puntuación al ítem 22 haciendo referencia al uso de imágenes a través de aplicaciones de software social. Por lo que podemos decir que nuestros jóvenes y nuestros 
mayores poseen una competencia de uso adecuada, aunque en el caso de las personas mayores se debe trabajar aún más.

Figura 3: Competencias de uso para la búsqueda y tratamiento de la información. Adultos mayores VS Jóvenes.

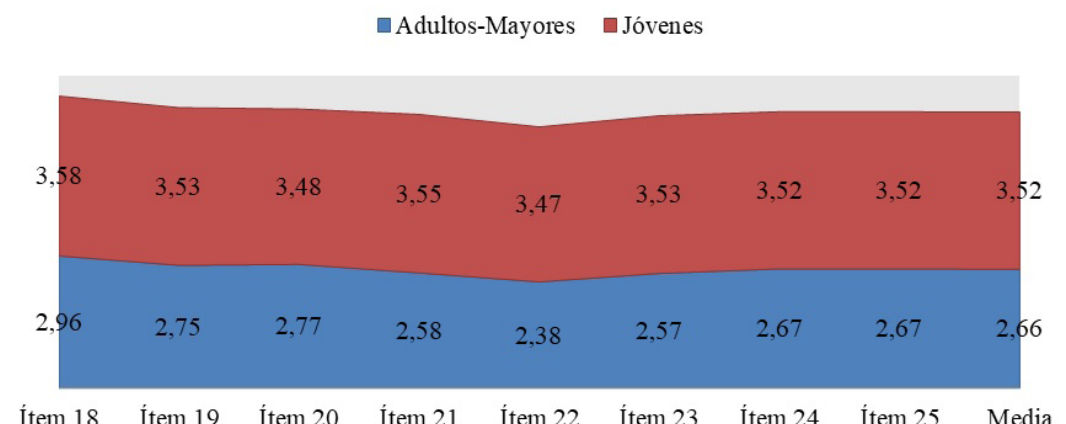

Fuente: los autores

En cuarto lugar, atendiendo a competencias interpersonales de su uso hemos obtenido una media total de 3,64 sobre 4 en los alumnos de Educación Primaria y 2,62 sobre 4 en los alumnos mayores. En el alumnado joven, al ítem 31 (hablar con algún compañero para solucionar el problema conjuntamente) es el que más puntuación se le ha otorgado, la menos puntuación se la otorgan al ítem 27 (exponer la duda en diferentes plataformas). En cambio, nuestros mayores otorgan una mayor puntuación al ítem 29, el cual hace referencia a reflexionar acerca de las dudas que se tienen antes de exponerlas a los demás. Sin embargo, otorgan una menor puntuación al ítem 26 consultando sus dudas mediante el correo institucional. Nuestros jóvenes y nuestros mayores poseen una competencia de interpersonal adecuada, aunque en el caso de las personas mayores se debe trabajar aún más. 
Figura 4: Competencias interpersonales de su uso. Adultos mayores VS Jóvenes.

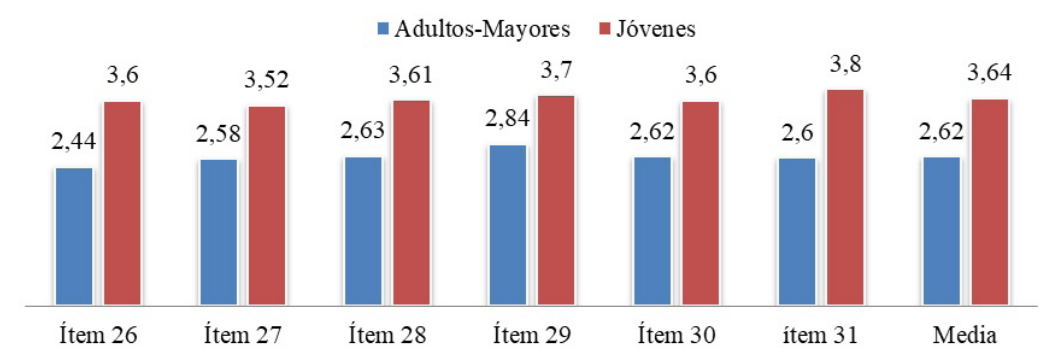

Fuente: los autores

\section{Discusión y conclusiones}

Este trabajo pone de manifiesto la importancia de conocer las diferencias entre la competencia digital en conocimiento y uso de las TIC en la comunicación social y aprendizaje colaborativo, competencias de uso para la búsqueda y tratamiento de la información, así como las competencias interpersonales de su uso entre jóvenes y adultos mayores de la Universidad de Granada. El trabajo con nuestros mayores en lo que a competencia digital se refiere debe ser una prioridad y un objetivo a conseguir como eje fundamental en la educación a lo largo de la vida. Culver y Jacobson (2012) explican que la alfabetización digital de las personas mayores para su inclusión en la sociedad actual debe promoverse para mejorar su calidad de vida durante el proceso de envejecimiento, ayudando a nuestros mayores a poseer una vida social más activa y participativa.

Los datos arrojados en nuestra investigación ponen de manifiesto reducir la brecha digital en nuestros mayores, siendo la esencia que ha caracterizado nuestra investigación. En este sentido procederemos a realizar unas conclusiones, que siguen las líneas de nuestro trabajo de investigación.

Haciendo referencia al consumo de la tecnología, los jóvenes poseen un consumo total del $100 \%$, por lo que el alumnado joven dispone de internet tanto en casa como en la facultad, conectándose habitualmente 
en ambos espacios. Sin embargo, en nuestros mayores no ocurre lo mismo, obtienen un 30\% asumiendo una escasa conexión habitual a internet.

La competencia digital en conocimiento y uso de las TIC en la comunicación social y aprendizaje colaborativo obtiene una media total de 3,66 sobre 4 en los alumnos de Educación Primaria y 1,50 sobre 4 en los alumnos mayores, destacamos que nuestros jóvenes poseen una competencia digital atendiendo al uso y conocimiento de las TIC bastante elevada, en cambio, nuestros mayores la poseen en menor medida.

En cuanto a las competencias de uso para la búsqueda y tratamiento de la información hemos hallado una media total de 3,52 sobre 4 en los alumnos de Educación Primaria y 2,66 sobre 4 en los alumnos mayores. Ambos colectivos, poseen una competencia de uso adecuada, aunque en el caso de las personas mayores se debe trabajar aún más.

Por último, atendiendo a las competencias interpersonales de su uso hemos obtenido una media total de 3,64 sobre 4 en los alumnos de Educación Primaria y 2,62 sobre 4 en los alumnos mayores, por lo que podemos decir que nuestros jóvenes y nuestros mayores poseen una competencia de interpersonal adecuada, aunque en el caso de las personas mayores se debe trabajar aún más.

Atendiendo a las cuatro grandes categorías de análisis, en general observamos una mayor competencia digital en los jóvenes que en los adultos mayores, por lo que aún queda mucho por trabajar para la inclusión de nuestro alumnado mayor en el uso de las Tecnologías de la Información y la Comunicación dentro y fuera de la universidad. Para ello, podemos poner en práctica proyectos de educación intergeneracional, para que los jóvenes transmitan dichos conocimientos a los alumnos de edad proyecta, creando un vínculo educativo entre distintas generaciones.

Como miembros de la sociedad tenemos la responsabilidad compartida de promover que los mayores que utilizan Internet saquen el mayor beneficio posible a la red y extiendan las funciones y ámbitos para los que la usan, y respecto a los que no lo hacen aún, facilitarles 
la formación necesaria y la motivación suficiente para que comiencen a utilizarla (MARTÍNEZ, CABECINHAS, y LOSCERTALES, 2011).

El sistema educativo actual debe contribuir a la eliminación del escalón generacional entre jóvenes y mayores en cuanto a competencia digital se refiere. Promoviendo la formación a lo largo de la vida y facilitando el acceso de los adultos mayores a las tecnologías, para un mejor uso y acceso a las mismas.

\section{Referencias}

ABAD, L. Diseño de programas de e-inclusión para alfabetización mediática de personas mayores. Comunicar, v. XXI, n. 42, p. 173-180, 2014.

ÁLVAREZ, G. T. Las redes sociales, recursos de autoformación de docentes. En J. Hernández, P. Fruscio, D. Sobrino y A Vázquez (coords). Experiencias educativas en las aulas del siglo XXI: innovación con TIC (pp. 373-375). Barcelona: Ariel, 2011.

ARRIDO-LORA, M.; BUSQUET DURAN, J.; MUNTÉ-RAMOS, R. De las TIC a las TRIC. Estudio sobre el uso de las TIC y la brecha digital entre adultos y adolescentes en España. Anàlisi. Quaderns de Comunicació i Cultura, n. 54, p. 44-57, 2016.

BALLESTEROS, J. C.; MEGÍAS, I. Jóvenes en la red: un selfie. Madrid: Centro Reina Sofía sobre Adolescencia y Juventud Fundación de Ayuda contra la Drogadicción (FAD), 2015. Disponible en: http://www.codajic.org/sites/www.codajic.org/files/jovenesen-la-red-selfie.pdf (consultado 14 de junio de 2018).

BENNETT, S.; MATON, K. Beyond the 'digital natives' debate: towards a more nuanced understanding of students' technology experiences. Journal of Computer Assisted Learning, v. 5, n. 26, p. 321-331, 2010.

BULLEN, M. M.; TANNIS; QAYYUM, A. Digital Learners in Higher Education: Generation is not the issue. Canadian Journal of Learning and Technology, v. 1, n. 37, p. $1-24,2011$.

CALVO, S.; SAN FABIÁN, J. L. Redes Sociales y Socialización Afectiva de las Personas Jóvenes: Necesidades Docentes en Educación Secundaria Obligatoria. REICE. Revista Iberoamericana sobre Calidad, Eficacia y Cambio en Educación, v. 16 , n. 2, p. 5-20, 2018. Disponible en: https://revistas.uam.es/index.php/reice/article/view/9232/9500

CASTELLS, M. La era de la información. Vol. 1. La sociedad red. (2ª edición), Madrid: Alianza, 2000.

CHÁVEZ, F.; CANTÚ, M. C.; RODRÍGUEZ C. Competencias digitales y tratamiento de información desde la mirada infantil. Revista electrónica de investigación educativa, v. 1 n. 18, p. 209-220, 2016.

COBADI. Competencias básicas digitales 2.0 de estudiantes universitarios. 2013. Disponible en: https://docs.google.com/forms/d/e/1FAIpQLSeu- 
wspXlrmhW1XUUQT1_FUz16OujR0JnquyGNvg2nepwEbR0w/

viewform?formkey=dHZhcFk5NUZEN1FDVjItX21XaGpmRnc6MQ\#gid=0

COLÁS-BRAVO, P.; GONZÁLEZ-RAMÍREZ, T.; DE PABLOS-PONS, J. Juventud y redes sociales: Motivaciones y usos preferentes. Comunicar. Revista Científica de Comunicación y Educación, v. 20, n. 40, p. 15-23. 2013.

CULVER, S.; JACOBSON, T. Alfabetización mediática como método para fomentar la participación cívica. Comunicar, n. 39, p. 73-80, 2012.

DAGHAN, G. Views of Students about Technology, Effects of Technology on Daily Living and Their Professional Preferences. Turkish Online Journal of Educational Technology-TOJET, v. 4 n. 16, p. 187-194, 2017.

DIARIO OFICIAL DE LA UNIÓN EUROPEA. Recomendación del Parlamento Europeo y del Consejo de 18 de diciembre de 2006 sobre competencias clave para el aprendizaje permanente. Bruselas: DOUE, 2006.

DOMÍNGUEZ, R. La sociedad del conocimiento y los nuevos retos educativos. Étic@ net, v. 8, n. 8, p. 1-19, 2009.

DUQUE, E. T. Adquisición de competencias digitales para la inclusión social. Opción, v. 9, n. 32, p. 610-630, 2016.

ESPINOZA, J. Reconfigurando el amor: Mediación tecnológica y relaciones afectivas. Question, v. 1 , n. 45, p. 86-96, 2015.

ESTEVE, F.; GISBERT, M. La competencia digital en la educación superior: instrumentos de evaluación y nuevos entornos. Enl@ce: Revista Venezolana de Información, Tecnología y Conocimiento, v. 10, n. 3, p. 29-43, 2013.

FAINHOLC, B. La lectura crítica en Internet: evaluación y aplicación de sus recursos. Comunicar, n. 26, p. 155-162, 2006.

FERRARI, A. Digital competence in practice: An analysis of frameworks. Luxembourg: Publications Office of the European Union, 2012. Disponible en: https://goo.gl/w8gdEj FREIXA, C. Generación XX. Teorías sobre la juventud en la era contemporánea. Revista Latinoamericana de Ciencias Sociales, Niñez y Juventud, v. 4, n. 2, p. 1-18, 2006.

GALLARDO, E. Hablemos de estudiantes digitales y no de nativos digitales. Revista de Ciències de l'Educació, p. 7-21, 2012.

GISBERT, M.; ESPUNY, C.; GONZÁLEZ, J. Incotic. Una herramienta para la @ utoevaluación diagnóstica de la competencia digital en la universidad. Revista de currículum y formación del profesorado, v. 1, n. 15, p. 75-90, 2011.

GONZÁLEZ-REYES, R. El internet como espacio de producción de capital social: una reflexión en torno a la idea de comunidad informal de aprendizaje. Revista mexicana de investigación educativa, v. 14, n. 40, enero-marzo, p. 175-190, 2009

GONZÁLEZ, J. E.; CINTA DE CID IBEAS, M. J.; GISBERT, M. INCOTICESO. Cómo autoevaluar y diagnosticar la competencia digital en la Escuela 2.0. Revista de Investigación Educativa, v. 2, n. 30, p. 287-302, 2012.

HEREDIA, N.; GARCÍA, E. Posibles riesgos del uso de las redes sociales en adolescentes. Revista de Estudios e Investigación en Psicología y Educación, n. 13, p. 11-15, 2017. 
HERNAND, A.; PHILLIPPI, A. El desarrollo de la competencia mediática en personas mayores: una brecha pendiente. Chasqui, v. 124, p. 12-18, 2013.

INSTITUTO DE MAYORES Y SERVICIOS SOCIALES (IMSERSO). Las personas mayores en España. Informe 2016, Madrid: Ministerio de Sanidad y Política Social: Instituto de Mayores y Servicios Sociales. 2017. Disponible en: http://www.imserso.es/ InterPresentl/groups/imserso/documents/binario/112017001_informe-2016-persona. pdf (consultado 14 de junio de 2018).

INSTITUTO NACIONAL DE ESTADÍSTICA. Encuesta sobre Equipamiento y Uso de Tecnologías de Información y Comunicación en los Hogares. Año 2017. Disponible en: http://www.ine.es/prensa/tich_2017.pdf

ITU. Facts And Figures ICT 2017. Geneva: International Telecommunication Union. 2017.

JARAMILLO, P.; HENNIG, C.; RINCÓN, Y. ¿Cómo manejan información los estudiantes de educación superior?: El caso de la Universidad de La Sabana, Colombia. Información, cultura y sociedad, v. 25, p. 117-143, 2011.

LANKSHEAR, C.; KNOBEL, M. Nuevos alfabetismo. Su práctica cotidiana y el aprendizaje en el aula. Madrid: Morata, 2008.

CANTABRANA, L., ESTEBANELL MINGUELL, M., Y TEDESCO, J. C. Inclusion and Social Cohesion in a Digital Society. RUSC. Universities and Knowledge Society Journal, v. 12, n. 2, p. 44-58, 2015.

LINNE, J. Estudiar en Internet 2.0 Prácticas de jóvenes universitarios de la Ciudad de Buenos Aires. Comunicación y Sociedad, n. 23, p. 195-213, 2015.

LIVINGSTONE, S.; HELSPER, E. Balancing Opportunities and Risks in Teenagers' Use of the Internet: the Role of On-line Skills and Internet Self-efficacy. New Media \& Society, n. 12, p. 309-329, 2010.

LLORENTE, C.; VIÑARÁS M.; SÁNCHEZ M. Mayores e Internet: La Red como fuente de oportunidades para un envejecimiento activo. Comunicar, n. 45, p. 29-36, 2015.

MARTÍNEZ, R.; CABECINHAS, R.; LOSCERTALES, F. University Senior Students on the Web. Comunicar, n. 37, p. 89-95, 2011.

PAVÓN, F. Tecnologías avanzadas: nuevos retos de comunicación para los mayores. Comunicar, n. 15, p. 133-139, 2000.

ROMÁN-GARCÍA, S.; ALMANSA-MARTÍNEZ, A.; CRUZ-DÍAZ, R. Adultos y mayores frente a las TIC. La competencia mediática de los inmigrantes digitales. Comunicar, n. 49, p. 101-109, 2016.

RUÍZ-CORBELLA, M.; DE-JUANAS, A. Redes sociales, identidad y adolescencia: nuevos retos educativos para la familia. Estudios sobre educación, n. 25, p. 95-113, 2013. SAHIN, C. Social Media Addiction Scale-Student Form: The Reliability and Validity Study. Turkish Online Journal of Educational Technology-TOJET, v. 17, n. 1, p. 169-182, 2018 
XAVIER, P.; CABECINHAS, R. Learning about Social Psychology by Researching on Computer Mediated Communication. IAMCR 2000 Conference. Singapore Proceedings, 2000.

YANG, S.; TUNG, C. J. Comparison of Internet Addicts and non-Addicts in Taiwanese High School. Computers in Human Behavior, n. 23, p. 79-96, 2007.

\section{Sobre los autores}

Ana Amaro Agudo - Profesora Ayudante Doctora del Dpto. Pedagogía de la Facultad de Ciencias de la Educación de la Universidad de Granada. En el presente artículo, la autora participó en la realización del marco teórico, la concepción del diseño de la investigación, en la recopilación e interpretación de datos, en la redacción del manuscrito y en la revisión del texto final.

Erika González García - Profesora Contratada Doctora del Dpto. Pedagogía de la Facultad de Ciencias de la Educación de la Universidad de Granada. En el presente artículo, la autora participó en la realización del marco teórico, la concepción del diseño de la investigación, en la recopilación e interpretación de datos, en la redacción del manuscrito y en la revisión del texto final.

Nazaret Martínez-Heredia - FPU (Formación del Profesorado Universitario) del Dpto. Pedagogía de la Facultad de Ciencias de la Educación de la Universidad de Granada. En el presente artículo, la autora participó en la realización del marco teórico, la concepción del diseño de la investigación, en la recopilación e interpretación de datos, en la redacción del manuscrito y en la revisión del texto final.

Data de submissão: 15/01/2020

Data de aceite: 06/03/2020 